\title{
Affine Quantization on the Half Line
}

\section{Laure Gouba}

The Abdus Salam International Centre for Theoretical Physics (ICTP), Trieste, Italy

Email: lgouba@ictp.it

How to cite this paper: Gouba, L. (2021) Affine Quantization on the Half Line. Journal of High Energy Physics, Gravitation and Cosmology, 7, 352-365.

https://doi.org/10.4236/jhepgc.2021.71019

Received: October 13, 2020

Accepted: January 26, 2021

Published: January 29, 2021

Copyright (c) 2021 by author(s) and Scientific Research Publishing Inc. This work is licensed under the Creative Commons Attribution International License (CC BY 4.0).

http://creativecommons.org/licenses/by/4.0/

\begin{abstract}
The similarity between classical and quantum physics is large enough to make an investigation of quantization methods a worthwhile endeavour. As history has shown, Dirac's canonical quantization method works reasonably well in the case of conventional quantum mechanics over $\mathbb{R}^{n}$ but it may fail in non-trivial phase spaces and also suffer from ordering problems. Affine quantization is an alternative method, similar to the canonical quantization, that may offer a positive result in situations for which canonical quantization fails. In this paper we revisit the affine quantization method on the half-line. We formulate and solve some simple models, the free particle and the harmonic oscillator.
\end{abstract}

\section{Keywords}

Classical Physics, Quantum Physics, Affine Quantization

\section{Introduction}

Although non-relativistic quantum mechanics stands as a well-established theory and a well experimentally test theory, the question of how to pass from classical to quantum theory and a better understanding of the relation between classical and quantum mechanics is still of particular interest. Indeed,

- Ongoing attempts to quantize general relativity where a definitive answer to the question of the correct quantum theory of gravitation is still missing;

- A quantization method that is able to take the nonlinear structure into account right from the outset is a useful tool to construct and study possible candidates for a theory of gravity;

- A better knowledge of quantization in a situation when physical systems satisfy constraints or boundary conditions is needed.

In physics, quantization is generally understood as a correspondence between a classical and a quantum theory. The question is how can we construct a quan- 
tum theory if a classical system is given? If we consider the quantum theory to be a more fundamental theory and classical mechanics to be only approximatively correct, the very concept of quantization seems pointless or appears to be ill-founded since it attempts to construct a correct theory from a theory which is only approximatively correct. For instance, there is the phenomenon of classical anomaly in the sense that if the quantization of some models gives the necessary quantum system, it may turn out that not all the quantum symmetry properties of the system are automatically reflected at the classical level [1]. There are quantum systems for which no classical counterpart exists: for example He-II superfluidity and some broad class of such systems can be found in the literature [2].

Quantum mechanics, like any other physical theory, classical mechanics, electrodynamics, relativity, thermodynamics, cannot be derived. The laws of quantum mechanics, expressed in mathematical form, are the results of deep physical intuition, as indeed, are all other physical theories. Their validity can only be checked experimentally. From this point of view, quantization is not a method for deriving quantum mechanics, rather is a way to understand the deeper physical reality which underlies the structure of both the classical and quantum mechanics and which unifies the two from geometrical perspectives.

It is conceptually very difficult to describe a quantum theory from scratch, without the help of a reference classical theory. The similarity between classical and quantum physics is large enough to make quantization a worthwhile approach. There is a certain mathematical richness in the various theories of quantization where the method does make sense. Quantization in its modern sense is therefore often understood as the construction of a quantum theory with the help of a classical reference, not necessarily as a strict mapping. Quantization is studied not only for the sake of novel predictions: it is equally rewarding to reproduce existing results in a more illuminating manner.

Originally P. A. M. Dirac introduced the canonical quantization in his 1926 doctoral thesis, The method of classical analogy for quantization [3]. The canonical quantization or correspondence principle is an attempt to take a classical theory described by the phase space variables, let's say $p$ and $q$, and a Hamiltonian $H(q, p)$ to define or construct its corresponding quantum theory. The following simple technique for quantizing a classical system is used. Let $q^{i}, p_{i}, i=1,2, \cdots, n$, be the canonical positions and momenta for a classical system with $n$ degrees of freedom. Their quantized counterparts $\hat{q}^{i}, \hat{p}_{i}, i=1,2, \cdots, n$, are to be realized as operators on the Hilbert space $\mathcal{H}=L^{2}\left(\mathbb{R}^{n}, d x\right)$ by the prescription

$$
\left(\hat{q}^{i} \psi\right)(x)=x^{i} \psi(x) ;\left(\hat{p}_{i} \psi\right)(x)=-i \hbar \frac{\partial}{\partial x^{i}} \psi(x) ; i=1,2, \cdots, n, x \in \mathbb{R}^{n} .
$$

This method is known as canonical quantization and is the basic method of quantization of a classical mechanics model [4] [5] [6]. More general quantities, such as the Hamiltonians, become operators according to the rule 


$$
H(p, q) \rightarrow \hat{H}(\hat{p}, \hat{q})
$$

an expression that may have ordering ambiguities [7] [8]. In which canonical coordinates system does such a quantization method works?

1) According to Dirac, replacing classical canonical coordinates by corresponding operators is found in practice to be successful only when applied with the dynamical coordinates and momenta referring to a cartesian system of axes and not to more general curvilinear coordinates.

2) Cartesian coordinates can only exist in a flat space.

3) The canonical quantization seems to depend on the choice of coordinates.

4) Beyond the ordering problem, one should keep in mind that $[\hat{q}, \hat{p}]=i \hbar I_{d}$ holds true with self-adjoint operators $\hat{q}, \hat{p}$, only if both have continuous spectrum $(-\infty,+\infty)$, and there is uniqueness of the solution, up to unitary equivalence (von Neumann).

There are two attitudes that may be taken towards this apparent dependence of the method of the canonical quantization on the choice of coordinates. The first view would be to acknowledge the cartesian character that is seemingly part of the method. The second view would be to regard it as provisional and seek to find a quantization formulation that eliminates this apparently unphysical feature of the current approaches.

The aim of eliminating the dependence on cartesian coordinates in the standard approaches is no doubt one of the motivations for several methods such as the geometric quantization [9] [10] [11] [12], the path integral quantization [13], the deformation quantization [10] [14] [15] [16] [17] [18], the Klauder-BerezinToeplitz quantization [19] [20] [21] [22]. There is no general theory of quantization presently available which is applicable in all cases, and indeed, often the techniques used to quantize has to be tailored to the problem in question.

As history has shown, Dirac's canonical quantization method works reasonably well in the case of conventional quantum mechanics over $\mathbb{R}^{n}$ due to the following reasons:

- The underlying configuration space $\mathbb{R}^{n}$ is so well behaved;

- When we try to quantize classical systems with phase spaces other than the cotangent bundle $T^{\star} \mathbb{R}^{n}$, the situation changes drastically;

- Already in classical mechanics, the phase spaces different from $T^{\star} \mathbb{R}^{n}$ require a more elaborated mathematical formalism;

- Global and topological aspects play a much bigger role in quantum theory than in classical physics.

Although quite successful in applications, the canonical quantization method has some severe shortcomings from a theoretical point of view. A number of questions arise in connection with the scheme of canonical quantization.

1) Let $Q$ be the position space manifold of the classical system and $q$ any point in it. Geometrically, the phase space of the system is the cotangent bundle $\Gamma=T^{\star} Q$. If $Q$ is linear, means $Q \sim \mathbb{R}^{n}$, then the replacement $q^{i} \rightarrow x^{i}$, 
$p_{j} \rightarrow-i \hbar \frac{\partial}{\partial x^{j}}$ works fine. But what happen if $Q$ is not linear?

2) How do we quantize observables which involve higher powers of $q^{i}, p_{j}$, as for example $f\left(q^{i}, p_{j}\right)=\left(q^{i}\right)^{n}\left(p_{j}\right)^{m}$ when $n+m \geq 3$ ?

3) How should we quantize more general phase spaces, which are the symplectic manifolds not necessarily cotangent bundles?

As we are currently interested in new developments in quantization methods [23] [24] [25], the goal of this paper is to highlight through simple models the benefits of affine quantization.

In Section (2), we revisit a method of quantization by J. R. Klauder, the affine quantization, then in Section (3) we formulate and solve the free particle and the harmonic oscillator. Concluding remarks are given in Section (4).

\section{Affine Quantization}

While in confinement due to COVID-19, our attention has been drawn on a recent published paper of J. R. Klauder on The benefits of Affine Quantization [26]. Our motivation is due to the fact that there is a difficulty with canonical quantization when it comes to configuration spaces other then $\mathbb{R}^{n}$. Consider, for example, a particle that is restricted to move on the positive real line. The configuration space is $\mathbb{Q}=\mathbb{R}^{+}$. It seems reasonable to use the position $q$ and momentum $\mathrm{p}$ as classical observables, which satisfy the usual commutations relations. However, when we try to represent these by operators $\hat{q} \equiv q$ and $\hat{p} \equiv-i \hbar \frac{\partial}{\partial q}$, it turns out that the momentum operator $\hat{p}$ is not self-adjoint on the Hilbert space $\mathcal{H}=L^{2}\left(\mathbb{R}^{+}, d q\right)$. Thus a straightforward application of Dirac's canonical quantization recipe is impossible.

Our goal is to apply the method of affine quantization to study the free particle and the harmonic oscillator that would serve as a test in order to study a toy model of a massive Klein Gordon field coupled to an harmonic oscillator at the the boundary considering the half line. In this section, we revisit the affine quantization method from some previous works of J. R. Klauder [27] [28] [29].

Let us start with a single degree of freedom, the classical phase space variables $p$ and $q$ are real satisfying a standard Poisson bracket

$$
\{q, p\}=1 \text {, }
$$

multiplying by $q$ Equation (3) we get

$$
q\{q, p\}=q,
$$

that is equivalent to $\{q, p q\}=q$, setting $d=p q$, we have

$$
\{q, d\}=q \text {. }
$$

The two variables $d$ and $q$ form a Lie-algebra and are worthy of consideration as new pair of classical variables even though they are not canonical coordinates. It is also possible to restrict $q$ to $q>0$ or $q<0$ consistent with $d$. The varia- 
ble $d$ acts to dilate $q$ and not to translate $q$ as the variable $p$ does.

For the case of the single degree of freedom above, the canonical quantization involves $\hat{q}$ and $\hat{p}$ which are self adjoint operators that satisfy the canonical commutation relation

$$
[\hat{q}, \hat{p}]=i \hbar I_{d} .
$$

From the canonical quantization, it follows that

$$
\hat{q}[\hat{q}, \hat{p}]=[\hat{q}, \hat{q} \hat{p}]=\left[\hat{q}, \frac{\hat{q} \hat{p}+\hat{p} \hat{q}}{2}\right] \equiv[\hat{q}, \hat{d}]=i \hbar \hat{q},
$$

where the dilation operator is define as $\hat{d} \equiv(\hat{p} \hat{q}+\hat{q} \hat{p}) / 2$ is self adjoint. The operator $\hat{d}$ is called the dilation operator because it dilates $\hat{q}$ rather than translates $\hat{q}$ as $\hat{p}$ does, in particular

$$
\mathrm{e}^{\frac{i q \hat{p}}{\hbar}} \hat{q} \mathrm{e}^{-\frac{i q \hat{p}}{\hbar}}=\hat{q}+q I_{d},
$$

while

$$
\mathrm{e}^{i \ln ((q)) \hat{d} / \hbar} \hat{q} \mathrm{e}^{-i \ln (|q|) \hat{d} / \hbar}=|q| \hat{q}=q|\hat{q}| .
$$

In the second relation in Equation (9), $q \neq 0$, and $q$ as well as $\hat{q}$ are normally chosen to be dimensionless. According to Equation (7), the existence of canonical operators guarantees the existence of affine operators. If $\hat{q}>0$ or $(\hat{q}<0)$, then the operator $\hat{p}$ cannot be made self-adjoint, however in that case, both of the operators $\hat{q}$ and $\hat{d}$ are self adjoint. As usual $\hat{q}$ and $\hat{p}$ are irreducible, but $\hat{q}$ and $\hat{d}$ are reducible. There are three inequivalent irreducible representations; one with $\hat{q}>0$, one with $\hat{q}<0$ and one with $\hat{q}=0$ and all three involve representations that are self-adjoint. The first two irreducible choices are the most interesting and, for the present, we focus on the choice $\hat{q}>0$.

\section{Testing Some Models}

\subsection{The Free Particle}

The simplest Hamiltonian one can envisage is the free particle on the half line. The Hamiltonian reads

$$
\mathcal{H}_{f}\left(x, p_{x}\right)=\frac{1}{2 m} p_{x}^{2},
$$

with $\left\{x, p_{x}\right\}=1, x>0$, in terms of the affine variables as described in Section (2) we may rewrite

$$
\mathcal{H}_{f}\left(x, d_{x}\right)=\frac{1}{2 m} d_{x} x^{-2} d_{x},
$$

where the variable $d_{x}$ is the dilation variable $d_{x}=p_{x} x$ and $\left\{x, d_{x}\right\}=x$. By mean of canonical quantization where the affine variables $x, d_{x}$ are respectively promoted to operators $\hat{x}, \hat{d}_{x}$ the corresponding Hamiltonian for the free particle reads 


$$
\hat{\mathcal{H}}_{f}\left(\hat{x}, \hat{d}_{x}\right)=\frac{1}{2 m} \hat{d}_{x}(\hat{x})^{-2} \hat{d}_{x},
$$

where $\hat{d}_{x}$ stands for the dilation operator and $\left[\hat{x}, \hat{d}_{x}\right]=i \hbar \hat{x}$. We have the representation $\hat{d} \equiv-i \hbar\left(x \partial_{x}+1 / 2\right)$ and $\hat{x} \equiv x$, with $x>0$. The time independent eigenvalue equation can be written as

$$
\left(\frac{1}{2 m} \hat{d}_{x}(\hat{x})^{-2} \hat{d}_{x}\right) \phi(x)=E \phi(x),
$$

that is equivalent to

$$
\left(-\frac{\hbar^{2}}{2 m} \frac{\mathrm{d}^{2}}{\mathrm{~d} x^{2}}+\frac{\hbar^{2}}{2 m} \frac{3}{4} x^{-2}\right) \phi(x)=E \phi(x) .
$$

We are then interested in solving the problem (14). If we divide by $-\frac{\hbar^{2}}{2 m}$ and setting $k^{2}=\frac{2 m E_{k}}{\hbar^{2}}$, where we assume $E_{k}>0$ and label $\alpha=3 / 4$, Equation (14) takes the form

$$
\phi_{k}^{\prime \prime}(x)=\left(\frac{\alpha}{x^{2}}-k^{2}\right) \phi_{k}(x) .
$$

Let's consider the change of variable variable $x=k^{-1} y$. The equation in (15) is rewritten in terms of the new variable $y$ as follows

$$
\phi^{\prime \prime}(y)=\left(\frac{\alpha}{y^{2}}-1\right) \phi(y) .
$$

Setting $\phi(y)=y^{1 / 2} \varphi(y)$ and reminding that $\alpha=3 / 4$, we obtain the ordinary differential equation

$$
\varphi^{\prime \prime}(y)+\frac{1}{y} \varphi^{\prime}(y)+\left(1-\frac{1}{y^{2}}\right) \varphi(y)=0,
$$

that is a variant of the Bessel's equation and the solution is defined by the Bessel function of order one, $J_{1}(x)$. A continuum of eigenfunctions exist for the problem (15), hence for problem (14) as

$$
\phi_{k}(x)=(k x)^{\frac{1}{2}} J_{1}(k x), E_{k}=\frac{k^{2} \hbar^{2}}{2 m}, k>0 .
$$

that are satisfying the important closure relation

$$
\int_{0}^{\infty} \phi_{k}(x) \phi_{k}(y) \mathrm{d} k=\delta(x-y), k>0 .
$$

since the order of the Bessel function is greater than $(-1 / 2)$. The details about that property can be found in reference [30].

\subsection{The Harmonic Oscillator}

We consider the one dimensional harmonic oscillator represented by the classical Hamiltonian

$$
H_{o}\left(x, p_{x}\right)=\frac{1}{2 m} p_{x}^{2}+\frac{1}{2} m \omega^{2} x^{2},
$$


where $\left(p_{x}, x\right) \in \mathbb{R} \times \mathbb{R}^{+}$, that means $x>0$, with $\left\{x, p_{x}\right\}=1$. Our aim is to test the affine quantization that has already been partially considered in [29]. Let us first determine the classical affine variables as in Section (2). We set $d_{x}=p_{x} x$, also called the dilation variable and the new variables called affine variables are $x$ and $d_{x}$ that satisfy the relation $\left\{x, d_{x}\right\}=x$. The Hamiltonian in Equation (20) can be then rewritten in terms of the affine variables as

$$
H_{a}\left(x, d_{x}\right)=\frac{1}{2 m} d_{x}\left(x^{-2}\right) d_{x}+\frac{1}{2} m \omega^{2} x^{2} .
$$

By mean of the affine quantization, the classical affine variables are promoted as operators

$$
d_{x} \rightarrow \hat{d}_{x} ; \quad x \rightarrow \hat{x}
$$

and the corresponding quantized Hamiltonian is

$$
\hat{H}_{a}\left(\hat{x}, \hat{d}_{x}\right)=\frac{1}{2 m} \hat{d}_{x}(\hat{x})^{-2} \hat{d}_{x}+\frac{1}{2} m \omega^{2} \hat{x}^{2} .
$$

The affine operators satisfies the commutation relations

$$
\left[\hat{x}, \hat{d}_{x}\right]=i \hbar \hat{x}
$$

and act as follows

$$
\hat{x} \psi(x, t)=x \psi(x, t) ; \quad x>0, \hat{x}>0,
$$

and

$$
\hat{d}_{x} \psi(x, t)=-i \hbar\left(x \frac{\partial}{\partial x}+\frac{1}{2}\right) \psi(x, t),
$$

where the wave functions are normalized

$$
\int_{0}^{\infty}|\psi(x, t)|^{2} \mathrm{~d} x=1 .
$$

We can then solve for the Schrödinger equation

$$
i \hbar \frac{\partial \psi(x, t)}{\partial t}=\hat{H}_{o} \psi(x, t)
$$

Since we are in presence of autonomous system we may set the Ansatz

$$
\psi(x, t)=\mathrm{e}^{-i t E / \hbar} \phi(x),
$$

and then the corresponding time-independant eigenvalue equation is

$$
\hat{H}_{a}\left(\hat{x}, \hat{d}_{x}\right) \phi(x)=E \phi(x),
$$

that is explicitly the equation

$$
\left(-\frac{\hbar^{2}}{2 m} \frac{\mathrm{d}^{2}}{\mathrm{~d} x^{2}}+\frac{3 \hbar^{2}}{8 m} \frac{1}{x^{2}}+\frac{1}{2} m \omega^{2} x^{2}\right) \phi(x)=E \phi(x),
$$

and we can rewrite as

$$
\left[-\frac{\mathrm{d}^{2}}{\mathrm{~d} x^{2}}+\frac{3}{4} \frac{1}{x^{2}}+\frac{m^{2} \omega^{2}}{\hbar^{2}} x^{2}\right] \phi(x)=\frac{2 m E}{\hbar^{2}} \phi(x) .
$$


For matter of simplication, let's set the following parameters

$$
\lambda=\frac{m \omega}{\hbar} ; \quad k^{2}=\frac{2 m E}{\hbar^{2}} ; \quad \alpha=\frac{3}{4},
$$

and Equation (32) becomes

$$
\left[-\frac{\mathrm{d}^{2}}{\mathrm{~d} x^{2}}+\lambda^{2} x^{2}+\frac{\alpha}{x^{2}}\right] \phi(x)=k^{2} \phi(x) .
$$

A standard asymptotic analysis for $x \rightarrow \infty, x \rightarrow 0$, requires the following Ansatz for the wave function

$$
\phi(x)=x^{\beta+1} \mathrm{e}^{-\frac{\lambda}{2} x^{2}} v(x),
$$

where the constant $\beta$ and $\alpha$ are related by $\alpha=\beta(\beta+1)$ and $v(x)$ is unknown function. The relation between $\alpha$ and $\beta$ gives two possible values of $\beta$ that are $\beta_{+}=+1 / 2$ and $\beta_{-}=-3 / 2$. We choose not to specify for the moment the values of $\beta$. From the Ansatz in Equation (35), an equation for the unknown function $v(x)$ is given by

$$
v^{\prime \prime}(x)+\left[2(\beta+1) x^{-1}-2 \lambda x\right] v^{\prime}(x)+\left[k^{2}-\lambda(2 \beta+3)\right] v(x)=0 .
$$

Making the change of variable $y=\lambda x^{2}$ in Equation (36), we obtain the differential equation

$$
y v^{\prime \prime}(y)+[(\beta+3 / 2)-y] v^{\prime}(y)+\left[\frac{k^{2}}{4 \lambda}-\frac{1}{4}(2 \beta+3)\right] v(y)=0 .
$$

The general solution of Equation (37) also known as Kummer's differential equation, can be expressed in terms of confluent hypergeometric functions

$$
\begin{aligned}
v(y)= & A_{1} F_{1}\left(\frac{1}{2}(\beta+3 / 2)-\frac{1}{2} \mu, \beta+3 / 2, y\right) \\
& +B y^{-(\beta+1 / 2)}{ }_{1} F_{1}\left(\frac{1}{2}(-\beta+1 / 2)-\frac{1}{2} \mu, \frac{1}{2}-\beta, y\right),
\end{aligned}
$$

where $\mu=k^{2} /(2 \lambda)$, and ${ }_{1} F_{1}(a, c, y)$ denotes the confluent hypergeometric function which has the following series representation

$$
{ }_{1} F_{1}(a, c, y)=1+\frac{a}{c} y+\frac{a(a+1)}{c(c+1)} \frac{y^{2}}{2 !}+\cdots
$$

Due to the asymptotic behavior of the confluent hypergeometric function given by

$$
{ }_{1} F_{1}(a, c, y) \sim \mathrm{e}^{y} y^{a-c},
$$

which implies divergencies of both of the terms in Equation (38) and then the impossibility to normalize the wave function, we impose the following conditions $\frac{1}{2}(\beta+3 / 2)-\frac{1}{2} \mu=-n$ or $\frac{1}{2}(-\beta+1 / 2)-\frac{1}{2} \mu=-n, n=1,2, \cdots$

For the first condition, that is $\frac{1}{2}(\beta+3 / 2)-\frac{1}{2} \mu=-n$, the eigenfunctions of 
Equation (32) have the form

$$
\phi_{n}(x)=A_{n} x^{\beta+1} \mathrm{e}^{-\frac{m \omega}{2 \hbar} x^{2}}{ }_{1} F_{1}\left(-n, \beta+3 / 2, \frac{m \omega}{\hbar} x^{2}\right),
$$

with the energy levels of the form $E_{n}=\hbar \omega(2 n+\beta+3 / 2), n=1,2, \cdots$, where the constants $A_{n}$ have to be determined using the normalization conditions. We have for $\beta=+1 / 2$, the solution

$$
\phi_{n}(x)=A_{n} x^{3 / 2} \mathrm{e}^{-\frac{m \omega}{2 \hbar} x^{2}} F_{1}\left(-n, 2, \frac{m \omega}{\hbar} x^{2}\right), E_{n}=2(n+1) \hbar \omega, n=1,2, \cdots
$$

The value of $\beta=-3 / 2$ is skipped as it leads to undefined confluent hypergeometric function.

For the second condition, that is $\frac{1}{2}(-\beta+1 / 2)-\frac{1}{2} \mu=-n$, the eigenfunctions of Equation (32) have the form

$$
\phi_{n}(x)=B_{n}\left(\frac{m \omega}{\hbar}\right)^{-(\beta+1 / 2)} x^{-\beta} \mathrm{e}^{-\frac{m \omega}{2 \hbar} x^{2}}{ }_{1} F_{1}\left(-n, \frac{1}{2}-\beta, \frac{m \omega}{\hbar} x^{2}\right),
$$

with $E_{n}=\left(2 n-\beta+\frac{1}{2}\right) \hbar \omega, n=1,2, \cdots$, where the constants $B_{n}$ have to be determined using the normalization conditions. For $\beta=-3 / 2$ we have

$$
\phi_{n}(x)=\left(\frac{m \omega}{\hbar}\right) B_{n} x^{3 / 2} \mathrm{e}^{-\frac{m \omega}{2 \hbar} x^{2}}{ }_{1} F_{1}\left(-n, 2, \frac{m \omega}{\hbar} x^{2}\right), E_{n}=2(n+1) \hbar \omega, n=1,2, \cdots
$$

The value of $\beta=+1 / 2$ leads in this case to an undefined confluent hypergeometric function.

The constant $A_{n}$ and $B_{n}$ in Equation (42) and respectively in Equation (44) are determined from the normalization condition

$$
\int_{0}^{\infty} \phi_{n}^{2}(x) \mathrm{d} x=1
$$

In order to compute the integral (45) for each case, we use a lemma which is a generalization of formula f6 in the book Quantum Mechanics by Landau and Lifshitz [30].

Lemma: For $\gamma>0$, and $m, n=0,1,2, \cdots$

$$
\int_{0}^{\infty} x^{2 \gamma-1} \mathrm{e}^{-\beta x^{2}}{ }_{1} F_{1}\left(-n ; \gamma, \beta x^{2}\right){ }_{1} F_{1}\left(-m ; \gamma, \beta x^{2}\right) \mathrm{d} x=\frac{1}{2} n ! \frac{\Gamma(\gamma)}{\beta^{\gamma}(\gamma)_{n}} \delta_{m n},
$$

where $\delta_{m n}=0$ for $m \neq n$ and 1 for $m=n$, and $(\gamma)_{n}$ is the Pochhammer symbol $(\gamma)_{n} \equiv \Gamma(\gamma+n) / \Gamma(\gamma)$.

The constants $A_{n}$ and $B_{n}$ respectively in Equations (42) and (44) are explicitly given by

$$
A_{n}=\left(\frac{m \omega}{\hbar}\right) \sqrt{\frac{2(2)_{n}}{n ! \Gamma(2)}}, \quad B_{n}=\sqrt{\frac{2(2)_{n}}{n ! \Gamma(2)}}, n=1,2, \cdots,
$$

which are simplified using $(2)_{n} \equiv \Gamma(2+n) / \Gamma(2)$ to 


$$
A_{n}=\left(\frac{m \omega}{\hbar}\right) \sqrt{2(n+1)}, \quad B_{n}=\sqrt{2(n+1)}
$$

To summarize the eigenfunctions and the eigenvalues of Equation (32) are respectively given by

$$
\begin{gathered}
\phi_{n}(x)=\sqrt{2(n+1)}\left(\frac{m \omega}{\hbar}\right) x^{3 / 2} \mathrm{e}^{-\frac{m \omega}{2 \hbar} x^{2}}{ }_{1} F_{1}\left(-n, 2, \frac{m \omega}{\hbar} x^{2}\right), \\
E_{n}=2(n+1) \hbar \omega, n=1,2, \cdots
\end{gathered}
$$

The eigenfunctions as we can see in Equation (49) satisfy the orthogonality conditions and are different from the ones of the full harmonic oscillator. They are similar to the ones of the family of harmonic oscillators to which a singular repulsion at the origin is added. The energy eigenvalues in Equation (50) are countably and equally spaced.

\section{Concluding Remarks}

We have revisited the procedure of affine quantization introduced by J. R. Klauder. Our motivation is to better understand this quantization method on nontrivial phase spaces where canonical quantization fails. We have tested the procedure for the simple case of the free particle and the case of the harmonic oscillator, both on the half-line.

For the case of the free particle in the upper half-line, the equivalent of the Hamiltonian in the affine coordinates turns out to be the case of a particle in a square inverse potential $V(x)=\alpha / x^{2}$. This kind of problem has been considered in the literature [31] [32] [33] and the case of $-1 / 4<\alpha<0$ has been explicitly discussed in [31]. There is a richness in the inverse square potential in quantum mechanics due to the connections to diverse physical phenomena [34] [35] [36].

For the case of the harmonic oscillator on the half-line, usually called the half-harmonic oscillator, the equivalent of the Hamiltonian in terms of the affine coordinates is the case of model with the potential $V(x)=(1 / 2) m \omega^{2} x^{2}+\alpha / x^{2}, x>0$, that is a well-known model in the literature. In fact, the family of quantum Hamiltonians known as spiked harmonic oscillators is given by the general Hamiltonian operator $H=-\mathrm{d}^{2} / \mathrm{d} x^{2}+x^{2}+\alpha / x^{2}$ acting on the Hilbert space $L^{2}(0, \infty)$. The name of the operator derives from the graphical shape of the full potential $V(x)=x^{2}+\alpha / x^{2}$ which shows a pronounced peak near the origin for $\alpha>0$. The spiked harmonic oscillator has drawn the attention of many authors since the publication of the pioneering paper of J. R. Klauder [38], four decades ago. It represents the simplest model of certain realistic interaction potentials in atomic, molecular and nuclear physics, and second and also due to its interesting intrinsic properties from the viewpoint of mathematical physics [39] [40]. The method used in Section (3) is similar to the one discussed in the problem of quantization of the systems with a position-dependent mass [41]. 
We have been curious about the possibility of getting back to canonical quantization from affine quantization. Is it possible to recover the solutions of the usual harmonic oscillator on the line from the solutions of the half harmonic oscillator? In order to analyse that question, we consider a positive real $b \geq 0$, where $-b<x$. In the situation of the half-line, the endpoint and the symmetry point coincide to 0 . Now we imagine the situation in which we save the symmetry point that remains 0 while the endpoint can move toward negative infinity. The situation is described by the Hamiltonian

$$
\hat{H}_{a}\left(\hat{x}, \hat{d}_{x}\right)=\frac{1}{2 m}\left(\left(\hat{d}_{x}+b \hat{p}_{x}\right)(\hat{x}+b)^{-2}\left(\hat{d}_{x}+b \hat{p}_{x}\right)\right)+\frac{1}{2} m \omega \hat{x}^{2},
$$

where $\hat{d}_{x}$ is the dilation operator defined in the previous sections and

$$
\left[\hat{x}, \hat{p}_{x}\right]=i \hbar,\left[\hat{x}, \hat{d}_{x}\right]=i \hbar \hat{x} .
$$

The corresponding time independent Schrödinger equation is given by

$$
\left[-\frac{\mathrm{d}^{2}}{\mathrm{~d} x^{2}}+\frac{3}{4} \frac{1}{(x+b)^{2}}+\frac{m^{2} \omega^{2}}{\hbar^{2}} x^{2}\right] \phi(x)=\frac{2 m E}{\hbar^{2}} \phi(x),
$$

When $b=0$, we recover the problem in Section (3), and when $b \rightarrow+\infty$ Equation (53) tends to the one of the harmonic oscillator on the line. While Equation (53) is not obvious to solve analytically, we guess that the limit $b \rightarrow+\infty$ leads from affine to canonical and should lead to usual even and odd about the symmetry point $x=0$ eigenfunctions. The study of this problem is presently under investigation as another example of how affine quantization deals with harmonic oscillator problems that canonical quantization cannot resolve [42].

We are also interested in considering more toy models for instance the case of the model that consists of a massive Klein Gordon field in $1+1$ dimensions restricted to the left half-line by a boundary and coupled to a harmonic oscillator at that boundary, thus introducing some additional degrees of freedom. The classical Hamiltonian is defined by

$$
\begin{aligned}
H= & \int_{-\infty}^{0} \mathrm{~d} x\left(\frac{1}{2} \pi(x, t)^{2}+\frac{1}{2}\left(\partial_{x} \phi(x, t)\right)^{2}+\frac{1}{2} \mu^{2} \phi(x, t)^{2}\right) \\
& +\beta \phi(0, t) q(t)+\frac{1}{2} m \omega^{2} q(t)^{2}+\frac{1}{2 m} p(t)^{2} .
\end{aligned}
$$

We hope to report on that problem soon [43].

As pointed out in reference [29], gravity does not fit well with canonical quantization and affine quantization may be an alternative procedure. So we are also interested in understanding how the affine quantization can help in quantum gravity.

\section{Acknowledgements}

L. Gouba would like to gratefully thank Professor John R. Klauder for the comments, the suggestions and the ongoing discussion on the paper and its extensions. 
L. Gouba would like to gratefully thank Professor Mikhail S. Plyushchay for useful and instructive discussions.

L. Gouba would like to thank the Family and the Friends for their presence and their constant support especially during the COVID-19 lockdown.

\section{Conflicts of Interest}

The author declares no conflicts of interest regarding the publication of this paper.

\section{References}

[1] Gamboa, J. and Plyushchay, S.M. (1998) Classical Anomalies for Spinning Particles. Nuclear Physics B, 512, 485-504. https://doi.org/10.1016/S0550-3213(97)00792-X

[2] Correa, F., Jakubský, V. and Plyushchay, S.M. (2009) Aharonov-Bohm Effect on AdS2 and Nonlinear Supersymmetry of Reflectionless Pöschl-Teller System. Annals of Physics, 324, 1078-1094. https://doi.org/10.1016/j.aop.2009.01.009

[3] Dirac, P.A.M. (1925) The Fundamental Equations of Quantum Mechanics. Proceedings of the Royal Society of London A: Mathematical, Physical and Engineering Sciences, 109, 642.

[4] Dirac, P.A.M. (1930) The Principles of Quantum Mechanics. Oxford University Press, Oxford, 157-162, 281-285.

[5] Weyl, H. (1950) The Theory of Groups and Quantum Mechanics. Dover Publishing Company Inc., New York, 274-275.

[6] von Neumann, J. (1955) Mathematical Foundations of Quantum Mechanics. Princeton University Press, Princeton.

[7] Born, M. and Jordan, P. (1925) On Quantum Mechanics. Zeitschrift für Physik, 34, 858-888. https://doi.org/10.1007/BF01328531

[8] Agarwal, B.S. and Wolf, E. (1970) Calculus for Functions of Noncommuting Operators and General Phase Space Methods in Quantum Mechanics. Physical Review D, 2, 2206-2225. https://doi.org/10.1103/PhysRevD.2.2206

[9] Weyl, H.K.H. (1927) Quantenmechanik und gruppentheorie. Zeitschrift für Physik, 46, 1-46. https://doi.org/10.1007/BF02055756

[10] Groenewold, H.J. (1946) On the Principles of Elementary Quantum Mechanics. Physica, 12, 405-460. https://doi.org/10.1016/S0031-8914(46)80059-4

[11] Souriau, J.M. (1966) Quantification Géometrique. Communications in Mathematical Physics, 1, 374.

[12] Kostant, B. (1970) Lecture Notes in Mathematics. Springer-Verlag, Berlin, Vol. 170, 87-208. https://doi.org/10.1007/BFb0079068

[13] Feynman, R.P. (1942) Principles of Least Action in Quantum Mechanics. Thesis Feynman.

[14] Bayen, F., Flato, M., Fronsdal, C., Lichnerowicz, A. and Sternheimer, D. (1978) Deformation Theory and Quantization I, II. Annals of Physics (NY), 111, 61-110, 111-151. https://doi.org/10.1016/0003-4916(78)90225-7

[15] Kontsevich, M. (1997) Deformation Quantization of Poisson Manifolds.

[16] Bordemann, M. and Waldmann, S. (1998) Formal GNS Construction and States in Deformation Quantization. Communications in Mathematical Physics, 195, 549-583. 
https://doi.org/10.1007/s002200050402

[17] Bordemann, M., Neumaier, N. and Waldmann, S. (1998) Homogeneous Fedosov Star Products on Cotangent Bundles I: Weyl and Standard Ordering with Differential Operator Representation. Communications in Mathematical Physics, 198, 363-396. https://doi.org/10.1007/s002200050481

[18] Dito, G. and Sternheimer, D. (2002) Deformation Quantization: Genesis, Developments and Metamorphoses.

[19] Klauder, J.R. (1967) Weak Correspondence Principle. Journal of Mathematical Physics, 8, 2392-2399. https://doi.org/10.1063/1.1705170

[20] Berezin, F.A. (1974) Quantization. Izvestiya Rossiiskoi Akademii Nauk. Seriya Matematicheskaya, 38, 1116-1175.

[21] Berezin, F.A. (1975) General Concept of Quantization. Communications in Mathematical Physics, 40, 153-174. https://doi.org/10.1007/BF01609397

[22] Coburn, L.A. (1994) Algebraic Methods in Operator Theory. Birkhäuser Springer, Boston, 101-108. https://doi.org/10.1007/978-1-4612-0255-4_12

[23] Gouba, L. (2019) New Trend in Quantization Method.

[24] Gouba, L. (2019) Beyond Coherent States Quantization. Journal of Physics: Conference Series, 1416, Article ID: 012012. https://doi.org/10.1088/1742-6596/1416/1/012012

[25] Gouba, L. (2018) Dirac's Method for the Two-Dimensional Damped Harmonic Oscillator in the Extended Phase Space. Mathematics, 6, 180.

https://doi.org/10.3390/math6100180

[26] Klauder, J.R. (2020) The Benefits of Affine Quantization. Journal of High Energy Physics, Gravitation and Cosmology, 6, 175-185. https://doi.org/10.4236/jhepgc.2020.62014

[27] Klauder, J.R. (2012) Enhanced Quantization: A Primer. Journal of Physics A: Mathematical and Theoretical, 45, Article ID: 285304. https://doi.org/10.1088/1751-8113/45/28/285304

[28] Klauder, J.R. (2016) When Canonical Quantization Fails, How to Fix It.

[29] Klauder, J. (2020) Quantum Gravity Made Easy. Journal of High Energy Physics, Gravitation and Cosmology, 6, 90-102.

[30] Arfken, G.B. and Werber, H.J. (2005) Mathematical Methods for Physics. 6th Edition, Elsevier, Amsterdam, 696.

[31] Landau, L.D. and Lifshitz, E.M. (1981) Quantum Mechanics: Non-Relativistic Theory. Pergamon, London.

[32] Paik, S.T. (2018) Teaching Renormalization, Scaling, and Universality with an Example from Quantum Mechanics. Journal of Physics Communications, 2, Article ID: 015016. https://doi.org/10.1088/2399-6528/aaa377

[33] Essin, A.M. and Griffiths, D.J. (2006) Quantum Mechanics of the $1 / \mathrm{x}^{2}$ Potential. American Journal of Physics, 74, 109-117. https://doi.org/10.1119/1.2165248

[34] Coon, S.A. and Holstein, B.R. (2002) Anomalies in Quantum Mechanics: The $1 / \mathrm{r}^{2}$ Potential. American Journal of Physics, 70, 513-519. https://doi.org/10.1119/1.1456071

[35] Camblong, H.E., Epele, L.N., Fanchiotti, H. and Garcia Canal, C.A. (2001) Quantum Anomaly in Molecular Physics. Physical Review Letters, 87, Article ID: 220402. https://doi.org/10.1103/PhysRevLett.87.220402

[36] Kraemer, T., et al. (2006) Evidence for Efimov Quantum States in an Ultracold Gas 
of Cesium Atoms. Nature, 440, 315-318. https://doi.org/10.1038/nature04626

[37] Kaplan, D.B., Lee, J.-W., Son, D.T. and Stephanov, M.A. (2009) Conformality Lost. Physical Review D, 80, Article ID: 125005. https://doi.org/10.1103/PhysRevD.80.125005

[38] Klauder, J.R. (1978) Continuous and Discontinuous Perturbations. Science, 199 735-740

[39] Aguilera-Navarro, V.C. and Guardiola, R. (1991) Nonsingular Spiked Harmonic Oscillators. Journal of Mathematical Physics, 32, 2135-2141. https://doi.org/10.1063/1.529186

[40] Hall, R.L., Saad, N. and von Keviczky, A.B. (2001) Spiked Harmonic Oscillator. Journal of Mathematical Physics, 43, 94-112. https://doi.org/10.1063/1.1418247

[41] Bravo, R. and Plyushchay, S.M. (2016) Position-Dependent Mass, Finite-Gap Systems. Physical Review D, 93, Article ID: 105023. https://doi.org/10.1103/PhysRevD.93.105023

[42] Gouba, L. The b-Problem for the Half Harmonic Oscillator. (In Preparation)

[43] Gouba, L. Affine Quantization of the Massive Klein Gordon Field Coupled to an Oscillator Harmonic at the Boundary. (In Preparation) 